\title{
Nanotomography based on double asymmetrical Bragg diffraction
}

\author{
Marco Stampanonia) \\ Institute of Biomedical Engineering, ETH and University of Zürich, Switzerland \\ and Swiss Light Source, Paul Scherrer Institute, Villigen, Switzerland \\ Gunther Borchert \\ Institut für Kernphysik, Forschungszentrum Jülich, Germany \\ Rafael Abela \\ Swiss Light Source, Paul Scherrer Institute, Villigen, Switzerland \\ Peter Rüegsegger \\ Institute of Biomedical Engineering, ETH and University of Zürich, Switzerland
}

(Received 7 October 2002; accepted 27 February 2003)

\begin{abstract}
Nondestructive investigations at ultrahigh spatial resolution can be carried out with synchrotron-based x-ray computer tomography. The performance of presently used detectors is restricted by scintillation properties, optical light transfer and charge coupled device granularity to a limit of $1 \mu \mathrm{m}$ spatial resolution and efficiency of a few percent. A recently developed detector, called Bragg magnifier, exploits double asymmetrical Bragg diffraction to efficiently produce hard $\mathrm{x}$-ray images with magnification factors up to $100 \times 100$ and pixel size of less than 200 $\times 200 \mathrm{~nm}^{2}$. Combining it with tomography, we obtained full volumetric information of a sample with spatial resolution well below $1 \mu \mathrm{m}$ in less than $40 \mathrm{~min}$. () 2003 American Institute of Physics. [DOI: $10.1063 / 1.1569428]$
\end{abstract}

Hard $\mathrm{x}$ rays $(>20 \mathrm{keV})$ have a large penetration depth and are well suited for the nondestructive investigation of opaque samples. Exploiting the high intensity of thirdgeneration synchrotron sources and combining hard $\mathrm{x}$-ray microscopy with tomographic techniques it is possible to obtain volumetric information of a specimen in the micrometer ${ }^{1-4}$ or even submicrometer ${ }^{5}$ scale with minimal sample preparation. This approach has caught the interest of medical $^{6,7}$ as well as materials science. ${ }^{8,9}$ The challenge to reach micrometer and submicrometer spatial resolution with hard $\mathrm{x}$ rays has spurred the development of detectors optimized for spatial resolutions to the detriment of efficiency. State-of-the-art devices, which convert $\mathrm{x}$ rays into visible light with a thin scintillator screen and project them onto a charge coupled device (CCD) with the help of suitable microscopic optics, claim $0.8 \mu \mathrm{m}$ resolution with an efficiency of $0.5 \%$ at $30 \mathrm{keV}{ }^{10}$ This requires large exposure time. Therefore, the scientific development aims to perform tomographic scans (usually this implies the acquisition of 500-1000 two-dimensional radiographic projections) with submicrometer resolution within minutes. Approaches with Fresnel zone plates ${ }^{11}$ or parabolic compound refractive lenses ${ }^{12}$ have demonstrated to reach submicrometer resolution $(0.3 \mu \mathrm{m})$ but they could be efficiently used only at energies well below $20 \mathrm{keV}$.

In this letter, we present an experiment in which a recently developed detector, called Bragg magnifier and described in detail in Ref. 13, has been used as an high-efficient hard x-ray microscope to acquire distortion free, magnified projection images of a sample with spatial resolution around $250 \mathrm{~nm}$. The experiment has been carried out at the Materials

${ }^{a)}$ Electronic mail: marco.stampanoni@psi.ch
Science (MS) beamline of the Swiss Light Source. So far the current of the electron beam as well as the wiggler gap have not yet reached the specified values. This caused a lack in the photon flux of a factor of 5-7. In spite of that, thanks to the high efficiency of the Bragg magnifier, we were able to collect a complete tomographic data set (500 projections) of a human bone trabecula in less than $40 \mathrm{~min}$ at $22.10 \mathrm{keV}$.

The Bragg magnifier performs two-dimensional magnification in the $\mathrm{x}$-ray regime exploiting the well-known principle of asymmetrical Bragg diffraction from two crossed flat crystals. The x-ray optics of the device consists of two asymmetrically cut silicon crystals mounted so that their reflection planes are perpendicular to each other, as shown in Fig. 1. The intensity distribution of the beam containing absorption information about the sample is collected by the Bragg mag-

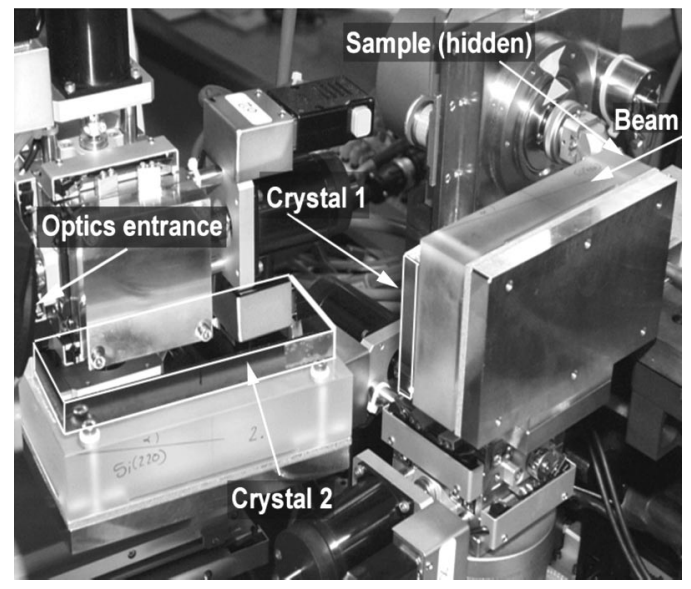

FIG. 1. X-ray optics of the Bragg magnifier: visible are both crystals fixed on their glass support and mounted on a high resolution goniometer. On the left side the entrance of the $1: 1$ optic is also visible. 
nifier optics and enlarged according to x-ray dynamical diffraction theory. In fact, for $\sigma$-polarized x rays as produced by wigglers of third-generation synchrotron facilities, the angular width of a beam diffracted by a nonabsorbing perfect crystal-for symmetric geometry and incident plane wave-is given by dynamical theory ${ }^{14,15}$ as $\omega_{S}=\left[2 / \sin \left(2 \theta_{B}\right)\right]\left(r_{0} \lambda^{2} / \pi V\right)\left|F_{h}\right| e^{-D W}$, where $r_{0}=2.818$ $\times 10^{-15} \mathrm{~m}$ is the classical electron radius, $\lambda$ is the wavelength of the incident radiation, $V$ is the volume of the unit cell, $\theta_{B}$ is the Bragg angle, $F_{h}$ is the crystal structure factor, and $e^{-D W}$ is the Debye-Waller factor. Defining the magnification factor as $m=\sin \left(\theta_{B}+\alpha\right) / \sin \left(\theta_{B}-\alpha\right)$ where $\alpha$ is the asymmetry angle, i.e., the angle between the crystal surface and its lattice planes, simple geometrical arguments lead to $S_{H}=|m| \cdot S_{O}$. According to Liouville's theorem, it follows that $\omega_{O}=|m| \cdot \omega_{H}$ where $\omega_{O}$ and $\omega_{H}$ are the angular divergences of the incident and diffracted beam, respectively. Quantitatively, dynamical theory states that $\omega_{O}=\sqrt{|m|} \cdot \omega_{S}$ and $\omega_{H}=1 / \sqrt{|m|} \cdot \omega_{S}$. It follows that if $|m|>1$ the range of total reflection for the emergent beam is $1 /|m|$ times smaller than that of the incident beam, while its spatial cross section is $|m|$ times greater. This allows to enlarge the radiographic projection of the sample.

$\mathrm{Si}(220)$ crystals with an asymmetry angle of $\alpha=8.0^{\circ}$ have been chosen as x-ray magnifying optics. The crystals have been cut with an angular accuracy of better than 1 arc min and have been fixed by optical contacting on a glass support. The crystal-glass unit is mounted on a steel flange which in turn is fixed to a double swivel. This system allows the crystal to be pitched and rolled with an angular reproducibility of $2 "$. The crossed swivels are mounted on a highresolution goniometer, which rocks the crystal with an angular resolution of $0.05^{\prime \prime}$ in order to cope with the very narrow rocking curve with $\omega_{0}=13.89^{\prime \prime}$ and $\omega_{H}=0.23^{\prime \prime}$ for an energy of $22.46 \mathrm{keV}$. The magnified x-ray image is collected by a high-efficiency position sensitive detector where it is first converted to visible light by a $35 \times 35 \mathrm{~mm}^{2} \mathrm{CsI}(\mathrm{Tl})$ scintillator of $300 \mu \mathrm{m}$ thickness, yielding a quantum efficiency of more than $90 \%$. Next the generated light is projected by a high-efficiency 1:1 tandem optics $(f=150 \mathrm{~mm}$, aperture $1 / 25$ ) onto a high performance CCD camera, featuring $2048 \times 2048$ pixels, 14 bit dynamic range, and multiple readout channels. ${ }^{16}$

In previous works ${ }^{13,17}$ it has been shown that the optics of the MS beamline can deliver a beam in the energy range of 21-23 keV with a divergence around 4" and that for these conditions, the Bragg magnifier produces hard x-ray images of excellent quality. Tuning the energy from 21.1 up to 22.75 $\mathrm{keV}$, and rocking both crystals accordingly we obtained magnification factors ranging from $20 \times 20$ to $100 \times 100$. Figure 2 shows a radiographic projection of a gold mesh (1500 periods/inch) acquired at $22.46 \mathrm{keV}$ with $10 \mathrm{~s}$ exposure time. For this energy the optics produce a magnification factor of $60 \times 60$ resulting in a theoretical pixel size of 233 $\times 233 \mathrm{~nm}^{2}$. Clearly visible are Fresnel fringes, principally due to the coherent nature of the beam. These patterns are more evident along the vertical direction because of the higher degree of coherence. As a consequence, and according to theoretical predictions, ${ }^{18}$ the images have to be interpreted as edge-enhanced projections. Further investigations of this

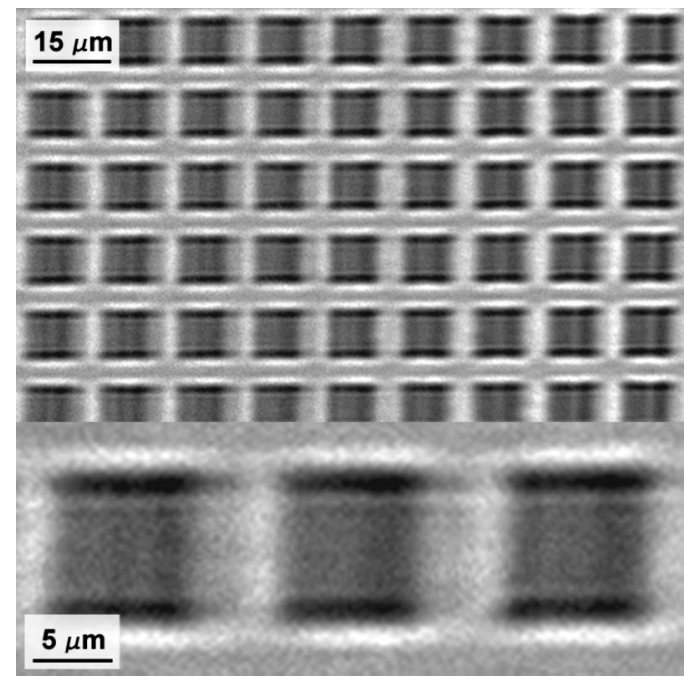

FIG. 2. Radiographic projection of a gold mesh with 1500 periods per inch acquired at $22.46 \mathrm{keV}$, corresponding to a magnification of $60 \times 60$. The inset shows a detail at a smaller scale.

phenomenon are on the way and will be discussed in a forthcoming article.

Tuning the energy to $22.35 \mathrm{keV}$ the Bragg magnifier produces a magnification of approximately $50 \times 50$ resulting in a theoretical pixel size of $280 \times 280 \mathrm{~nm}^{2}$. Thanks to the size of the crystals $\left(50 \times 150 \mathrm{~mm}^{2}\right)$, the optics cover a field of view of $575 \times 575 \mu \mathrm{m}^{2}$ which allows the investigation of a broad variety of samples. With these settings, we acquired a radiographic projection of a human bone trabecula, depicted in Fig. 3. Although a 300- $\mu$ m-thick unstructured $\mathrm{CsI}(\mathrm{Tl})$ scintillator has been used (providing a quantum efficiency of more than $90 \%$ ), single osteocytes as well as structures of less than $1 \mu \mathrm{m}$ can easily be detected. The unstructured $\mathrm{CsI}(\mathrm{Tl})$ scintillator will be replaced by a structured one ${ }^{19}$ providing an even higher spatial resolution by preserving the same high efficiency. Reducing the energy of the $\mathrm{x}$-ray beam to $22.10 \mathrm{keV}$, a magnification of $40 \times 40$ has been obtained. This corresponds to a theoretical pixel size of $350 \times 350 \mathrm{~nm}^{2}$ and a field of view of $715 \times 715 \mu \mathrm{m}^{2}$. The

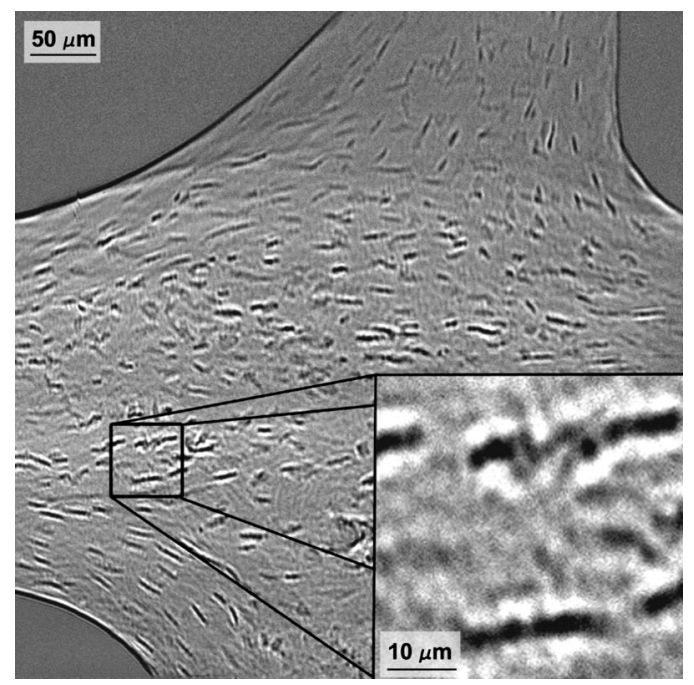

FIG. 3. Radiography of a human bone trabecula. Magnification factor is $50 \times 50$ and field of view is $575 \times 575 \mu \mathrm{m}^{2}$. The inset clearly shows osteocytes. 


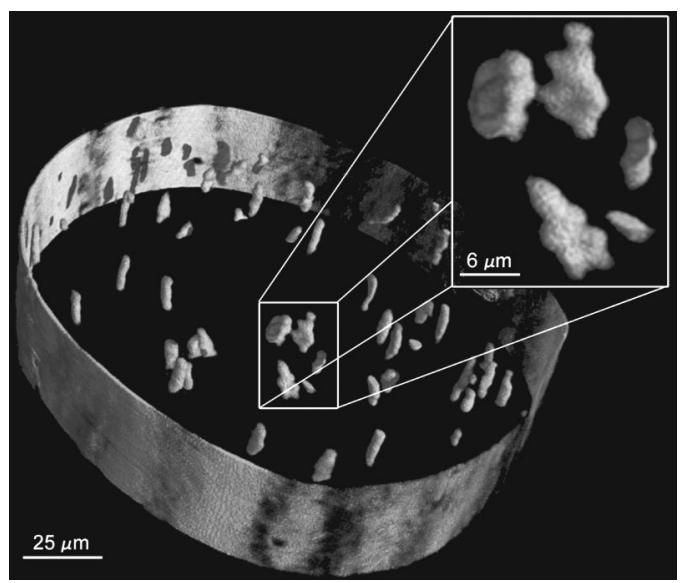

FIG. 4. Three-dimensional rendering of the trabecula in edge-enhanced modus. Voxel size $350 \times 350 \times 350 \mathrm{~nm}^{3}$ at $22.10 \mathrm{keV}$. The inset shows single osteocytes.

larger field of view easily accommodates the whole trabecula, even during a $[0, \pi]$ rotation along the main axis. In addition, the lower magnification factor compensates the reduced flux due to the suboptimal settings of electron beam current and wiggler gap. With this configuration, 500 twodimensional projections of the human trabecula have been acquired in equidistant angular intervals from $0^{\circ}$ to $180^{\circ}$. The exposure time was $5 \mathrm{~s}$ for each projection resulting in a total scan time of approximately $40 \mathrm{~min}$. After every 25 projections, the sample was moved out of the field of view to acquire a flat field image. The reproducibility of the translation stages as well as the run-out of the rotation axis (airbearing) were better than $100 \mathrm{~nm}$ while the wobble was less than $100 \mathrm{nrad}$. Flat and dark field corrected data have been reconstructed with a standard filtered-backprojection algorithm. ${ }^{20}$ In this way, thanks to the edge-enhancement effect, single osteocytes could be easily segmented, as impressively shown in Fig. 4.

The Bragg magnifier has been successfully operated at energy between 21.10 and $22.75 \mathrm{keV} .{ }^{13}$ This means that magnification factors up to $100 \times 100$ can be obtained leading to theoretical pixel sizes of $140 \times 140 \mathrm{~nm}^{2}$. When both electron beam current as well as wiggler gap will reach their specified values, sufficient flux will be available in order to efficiently perform nanotomography with voxel's size around $100 \mathrm{~nm}^{3}$.

The authors thank B. Patterson, D. Vermeulen, and M. Lange for their generous help in setting up the beamline. This work has been supported by the ETH Board.

${ }^{1}$ U. Bonse and F. Bush, Prog. Biophys. Mol. Biol. 65, 133 (1996).

${ }^{2}$ R. Lee, B. Lai, W. Yun, D. C. Mancini, and Z. Cai, Proc. SPIE 3149, 257 (1997)

${ }^{3}$ B. A. Dowd, G. H. Campbell, R. B. Marr, V. V. Nagarkar, S. V. Tipnis, L. Axe, and D. P. Siddons, Proc. SPIE 3772, 224 (1999).

${ }^{4}$ T. Weitkamp, C. Raven, and A. Snigierv, Proc. SPIE 3772, 311 (1999).

${ }^{5}$ C. G. Schroer, B. Benner, T. F. Günzler, M. Kuhlmann, B. Lengeler, C. Rau, T. Weitkamp, A. Snigirev, and I. Snigireva, Proc. SPIE 4503, 23 (2002).

${ }^{6}$ M. Salome, F. Peyrin, P. Cloetens, C. Odet, A. M. Laval-Jaentet, J. Baruchel, and P. Spanne, Med. Phys. 26, 2194 (1999).

${ }^{7}$ S. Nuzzo, F. Peyrin, P. Cloetens, J. Baruchel, and G. Boivin, J. Bone Miner. Res. 17, 1372 (2002).

${ }^{8}$ R. H. Mathiesen, L. Arnberg, F. Mo, T. Weitkamp, and A. Snigirev, Phys. Rev. Lett. 83, 5062 (1999).

${ }^{9}$ P. Cloetens, M. Pateyron Salome, J. Y. Buffiere, G. Peix, J. Baruchel, F. Peyrin, and M. Schlenker, J. Appl. Phys. 81, 5878 (1997).

${ }^{10}$ A. Koch, C. Raven, P. Spanne, and A. Snigirv, J. Opt. Soc. Am. A 15, 1940 (1998).

${ }^{11}$ B. Lai, W. Yun, Y. Xiao, D. Legnini, Z. Cai, A. Krasnoperova, F. Cerrina, E. Di Fabrizio, L. Grella, and M. Gentili, Rev. Sci. Instrum. 66, 2287 (1995).

${ }^{12}$ C. Schroer, J. Meyer, M. Kuhlmann, B. Benner, T. F. Günzler, B. Lengeler, C. Rau, T. Weitkamp, A. Snigirev, and I. Snigireva, Appl. Phys. Lett. 81, 1527 (2002).

${ }^{13}$ M. Stampanoni, G. L. Borchert, R. Abela, and P. Rüegsegger, J. Appl. Phys. 92, 7630 (2002).

${ }^{14}$ W. H. Zachariasen, Theory of X-Ray Diffraction in Crystals (Wiley, New York, 1945).

${ }^{15}$ A. Authier, Dynamical Theory of X-Ray Diffraction (Oxford University Press, New York, 2001)

${ }^{16}$ M. Stampanoni, G. L. Borchert, P. Wyss, R. Abela, B. Patterson, S. Hunt, D. Vermeulen, and P. Rüegsegger, Nucl. Instrum. Methods Phys. Res. A 491, 291 (2002)

${ }^{17}$ M. Stampanoni, G. L. Borchert, R. Abela, B. Patterson, D. Vermeulen, P. Rüegsegger, and P. Wyss, Acta Phys. Pol. B 33, 463 (2002).

${ }^{18}$ R. D. Spal, Phys. Rev. Lett. 86, 3044 (2002).

${ }^{19}$ V. V. Nagarkar, T. K. Gupta, S. R. Miller, Y. Klugerman, M. R. Squillante, and G. Entine, IEEE Trans. Nucl. Sci. 45, 492 (1998).

${ }^{20}$ A. C. Kak and M. Slaney, Principles of Computerized Tomographic Imaging (IEEE, New York, 1988). 